\title{
AN EXTREMELY HIGH 'Q'UEUE VALUE APPLIED TO SEISMOLOGICAL MEETINGS
}

Anonymous ${ }^{1}$

(perhaps Hunger and Tooth)

Abstract. Recent observations of the mysterious astronomical body IAU 123 indicate that the ' $Q$ 'ueue value for astronomy symposia can approach 100120, with very rapid growth and slow exponential decay. Number density fluctuations, with a frequency of $11.56 \mu \mathrm{Hz}$ and extreme phase coherence have been detected outside the main cafeteria. The long period and high ' $Q$ ' suggest that this may be represented by a g-mode of low radial order.

A stabilized Fabry-Perot etalon has been used to measure the low-level motions, with propagation velocities of order $0.01 \mathrm{~cm} \mathrm{~s}^{-1}$. Although this is well below the local Alfven speed, there can still arise significant shock effects (e.g. upon discovery of liver loaf as the main course).

The observed cafeteria oscillation spectrum is still incomplete. For instance, if ice cream were offered at the end of the line, we would expect to detect the well-known 'a la'-mode predicted by Baskin and Robbins.

We hope that future studies of astronomical $Q$ 's may lead to a better understanding of solar-type astronomers' resonant cavities. morning.

${ }^{1}$ Their emaciated skeletons were found outside the cafeteria early this 